\title{
QUEEN'S
UNIVERSITY
BELFAST
}

\section{Dependence of laser-driven coherent synchrotron emission efficiency on pulse ellipticity and implications for polarization gating}

\begin{abstract}
Yeung, M., Dromey, B., Cousens, S., Dzelzainis, T., Kiefer, D., Schreiber, J., Bin, J. H., Ma, W., Kreuzer, C., Meyer-Ter-Vehn, J., Streeter, M. J. V., Foster, P. S., Rykovanov, S., \& Zepf, M. (2014). Dependence of laserdriven coherent synchrotron emission efficiency on pulse ellipticity and implications for polarization gating.
\end{abstract} Physical Review Letters, 112(12). https://doi.org/10.1103/PhysRevLett.112.123902

Published in:

Physical Review Letters

Document Version:

Publisher's PDF, also known as Version of record

Queen's University Belfast - Research Portal:

Link to publication record in Queen's University Belfast Research Portal

Publisher rights

Copyright American Physical Society 2014

\section{General rights}

Copyright for the publications made accessible via the Queen's University Belfast Research Portal is retained by the author(s) and / or other copyright owners and it is a condition of accessing these publications that users recognise and abide by the legal requirements associated with these rights.

Take down policy

The Research Portal is Queen's institutional repository that provides access to Queen's research output. Every effort has been made to ensure that content in the Research Portal does not infringe any person's rights, or applicable UK laws. If you discover content in the Research Portal that you believe breaches copyright or violates any law, please contact openaccess@qub.ac.uk. 


\title{
Dependence of Laser-Driven Coherent Synchrotron Emission Efficiency on Pulse Ellipticity and Implications for Polarization Gating
}

\author{
M. Yeung, ${ }^{1, *}$ B. Dromey, ${ }^{1, \dagger}$ S. Cousens, ${ }^{1}$ T. Dzelzainis, ${ }^{1}$ D. Kiefer, ${ }^{2,5}$ J. Schreiber, ${ }^{2,5}$ J. H. Bin, ${ }^{2,5}$ W. Ma, ${ }^{2,5}$ C. Kreuzer, ${ }^{5}$ \\ J. Meyer-ter-Vehn, ${ }^{2}$ M. J. V. Streeter, ${ }^{3}$ P. S. Foster, ${ }^{4}$ S. Rykovanov, ${ }^{5, \$}$ and M. Zepf ${ }^{1,6}$ \\ ${ }^{1}$ Department of Physics and Astronomy, Queen's University Belfast, Belfast BT7 1NN, United Kingdom \\ ${ }^{2}$ Max-Planck-Institut für Quantenoptik, Hans-Kopfermann-Straße 1, D-85748 Garching, Germany \\ ${ }^{3}$ Blackett Laboratory, Imperial College London, London SW7 2BZ, United Kingdom \\ ${ }^{4}$ Central Laser Facility, STFC Rutherford Appleton Laboratory, Chilton, Didcot OX11 OQX, United Kingdom \\ ${ }^{5}$ Fakultät für Physik, Ludwig-Maximilians-Universität München, D-85748 Garching, Germany \\ ${ }^{6}$ Helmholtz-Institut Jena, Fröbelstieg 3, 07743 Jena, Germany
}

(Received 8 December 2013; published 26 March 2014)

\begin{abstract}
The polarization dependence of laser-driven coherent synchrotron emission transmitted through thin foils is investigated experimentally. The harmonic generation process is seen to be almost completely suppressed for circular polarization opening up the possibility of producing isolated attosecond pulses via polarization gating. Particle-in-cell simulations suggest that current laser pulses are capable of generating isolated attosecond pulses with high pulse energies.
\end{abstract}

DOI: 10.1103/PhysRevLett.112.123902

PACS numbers: 42.65.Ky, 52.27.Ny, 52.35.Mw

Ultrahigh intensity laser interactions with overdense plasma surfaces can produce coherent beams of extremeultraviolet (XUV) and $\mathrm{x}$-ray radiation via high harmonic generation (HHG) with remarkable spatial properties [1], high brightness [2], and attosecond (as) temporal duration [3] through a variety of processes and interaction geometries. Beyond the well-known processes such as coherent wake emission (CWE, dominant for weakly relativistic interactions [4]) and the relativisitic oscillating mirror (ROM, dominant in the strongly relativistic regime [5]), coherent synchrotron emission (CSE) [6,7] has recently come to the fore. CSE is characterized by its distinctive spectral shape (slow decay to higher frequencies up to the cutoff) and correlates with the formation of a distinct highdensity, nm-scale electron bunch observed in simulations. While coherent synchrotron emission can become dominant even at oblique incidence in reflection (normally associated with ROM), it is the only efficient mechanism in normal incidence in transmission [7] and has been shown to be a source of highly energetic XUV pulses.

In common with other HHG techniques, the temporal substructure of CSE is characterized by a train of attosecond pulses. This temporal substructure has given rise to the generation of isolated attosecond pulses for applications in attoscience [8], where low energy attosecond pulses are generated at moderate intensities when a femtosecond laser interacts with a gaseous medium [9]. Isolating a single attosecond pulse can be achieved either with extremely short laser pulses which exhibit a single dominant laser cycle and hence a single dominant attosecond pulse in the temporal structure. Alternatively, the strong ellipticity dependence of HHG in gaseous media is frequently exploited to achieve the generation of a single, isolated attosecond pulse. This is achieved by generating pulses with a complex polarization state [10], which exhibits strong ellipticity throughout the pulse, with the exception of one linearly polarized cycle at peak intensity where the pulse switches from left to right circular (or vice versa). For all optical cycles with significant ellipticity the generation of XUV harmonics is suppressed, and hence only a single attosecond pulse is emitted.

Producing highly energetic attosecond pulses requires converting a high energy laser pulse efficiently to coherent XUV radiation, making schemes that can exploit the relativistic intensities achieved when a petawatt class laser is focused with typically available focusing optics attractive. The challenge is then to achieve isolated attosecond pulses and therefore to understand the ellipticity dependence of the generation mechanism.

We investigate for the first time the polarization dependence of coherent synchrotron emission and observe the almost complete suppression of the harmonic emission for circular polarization during a relativistic laser-plasma interaction. This is the first time polarization controlled suppression of a HHG process has been observed for high energy laser pulses with relativistic intensities-key to producing bright, energetic attosecond pulses. Our particlein-cell (PIC) simulations demonstrate that the observed ellipticity scaling is sufficient to allow the production of a single attosecond pulse by implementing polarization gating techniques on currently available laser systems.

CSE relies on the formation of high-density, nanometer scale electron bunches (nanobunches) during the interaction of an ultraintense laser with an overdense plasma. The first numerical investigations reported on the formation of such bunches under oblique incidence interactions and 
the CSE spectra emitted in the specularly reflected direction [6]. While this mechanism exhibits a favorable efficiency scaling towards shorter wavelengths, it is difficult to observe in isolation for a reflection geometry due to the competing HHG mechanisms such as ROM and CWE. Recent experimental work, however, has demonstrated the unambiguous observation of CSE from subwavelength thick foils in a normal-incidence transmission geometry [7]. Simulations of the interaction identified the nanobunches of electrons pulled from the target surface by the $\mathbf{v} \times \mathbf{B}$ component of the Lorentz force as the origin of the radiation. The velocity $\mathbf{v}$ in this case is the transverse velocity that is predominantly accumulated in the electric field of the laser. When this velocity is relativistic (which occurs when the laser vector potential $a_{0}=I \lambda^{2} / 1.37 E 18 \mathrm{~W} \mathrm{~cm}^{-2} \mu \mathrm{m}^{2}>1$ ) then the laser's magnetic field $\mathbf{B}$ can drive the electrons in the longitudinal direction with a large amplitude. These bunches are then subsequently driven back into the plasma with speeds close to that of light. As they approach the target surface, their large forward velocity, combined with a transverse acceleration provided by the laser electric field, cause the nanobunches to emit CSE. When CSE is produced with femtosecond pulses [11], the temporal structure consists of only a small number of attosecond bursts, allowing polarization gating schemes such as interferometric polarization gating (IPG) [12] or the generalized double optical gating method [13] to be considered to isolate a single attosecond pulse.

The ellipticity dependence of CSE was investigated using the Astra Gemini laser system. The laser produced 50 fs duration pulses which, after contrast enhancement by a double plasma mirror setup and focusing by an $f / 2$ off-axis parabola to a near-diffraction-limited spot, yielded intensities of $\approx 5 \times 10^{20} \mathrm{~W} \mathrm{~cm}^{-2}$. The contrast after the plasma mirror system was $\approx 1: 10^{-10}$ at 10 ps before the pulse peak.

The transmitted harmonic radiation was detected on an XUV spectrometer consisting of a $270 \mu \mathrm{m}$ vertical slit placed $30 \mathrm{~cm}$ from the laser focus followed by a 1000 lines $/ \mathrm{mm}$ freestanding gold transmission grating. The dispersed spectrum is detected by a microchannel-plate (MCP) detector [see Fig. 1(a)].

A mica zero-order quarter-wave plate was used to generate a circularly polarized pulse, the effect of which can be seen in Fig. 1(b), which compares the spectra for a linearly (shaded area) and a circularly polarized (solid black line) driving pulse from a $300 \mathrm{~nm}$ parylene- $N$ foil. Harmonic orders up to the 15 th were clearly observed while the spectral resolution of the diagnostic limited the observation of higher orders. Of most interest from this graph is the almost complete suppression of the signal to the level of the detection noise floor in the case of circular polarization. We estimate the energy contained in the 9th harmonic to be within the $\mu \mathrm{J}$ range; however, without knowledge of the beam divergence a more precise value cannot be inferred at this point. However, this energy range

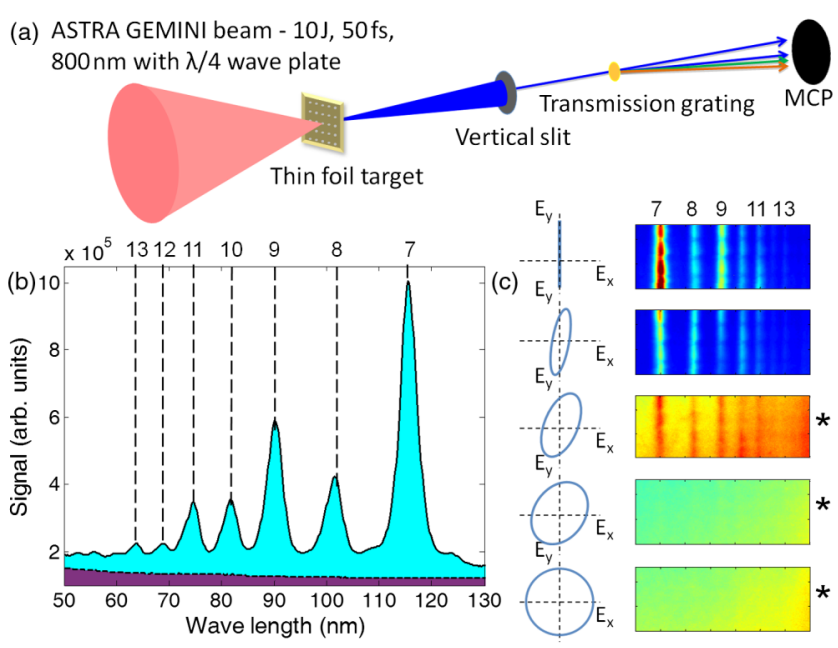

FIG. 1 (color online). (a) Sketch of the experimental setup and raw data showing the effect of pulse ellipticity. The laser is focused by an $f / 2$ off-axis parabola onto the ultrathin foil target. The transmitted XUV light is analyzed by passing it through a narrow slit or pinhole followed by a 1000 lines $/ \mathrm{mm}$ transmission grating which spectrally disperses the light onto an MCP detector. (b) XUV spectra-the shaded areas below the curves show the difference in signal for the linear driver (upper solid curve) and a circular driver (lower dotted curve). (c) Ellipticity scaling - the raw MCP images are shown for each ellipticity setting as indicated by the adjacent electric field vector plot. The lower three images indicated by an asterisk have been shown at a 5 times reduced color-map scale.

is comparable with efficiencies from previous measurements of this transmitted harmonic emission [7] but considerably lower than that expected for ideal cases of CSE in reflection [6]. A possible explanation for the discrepancy is the fact that the nanobunch density depends sensitively on the precise interaction parameters, particularly the laser contrast and preplasma scale length [6], which were not scanned systematically on this occasion.

Also of note here is the observation of even ordered harmonics, despite the interaction occurring at normal incidence. In the ideal one-dimensional case one would not expect to see even harmonics at all as the $v \times B$ force drives the generation mechanism twice per laser cycle [5]. However, it has been observed in 2D simulations of intense laser-foil interactions that off axis, the electron bunches are driven once per cycle (see, for example, Fig. 7 of Ref. [14]). This is due to electron bunches formed in alternating half cycles of the laser gaining transverse momentum from the laser electric field in opposite directions. The periodicity of these bunches would permit the generation of even harmonics and would explain these observations.

Figure 1(c) shows the raw transmitted images of the spectra for a sequence of shots on identical targets, but for a variety of ellipticity values. The Lissajous curves show the corresponding polarization ellipses obtained by rotation of the quarter wave plate. Enhancement of the lower images 
clearly shows that the harmonic emission is almost completely suppressed and that the drop in efficiency with driver ellipticity is rapid. In Fig. 2 the background subtracted and spatially integrated harmonic signal is plotted against the pulse ellipticity. It can be seen that for circular polarization the transmitted harmonic signal is almost reduced to the noise floor of the spectrometer which is determined by integrating the signal for a shot with no target, providing ideal conditions for applying polarization gating to CSE. In terms of the interaction physics, the substantial reduction in efficiency can be understood from considering the forces acting upon the target. At normal incidence the only oscillatory component of the force normal to the interaction surface is the $v \times B$ term. For circular polarization both magnitude and direction of the force become constant over a laser cycle for sufficiently slowly varying intensity envelope, hence preventing the formation of nanobunches and the emission of CSE. The absence of an oscillatory force component normal to the interaction surface suppresses all known HHG processes more generally and a similar suppression has been predicted for ROM harmonics also [5,17]. To our knowledge, this is the first experimental observation of near complete HHG suppression in a relativistic regime using circular polarization. As a comparison, measurements of the efficiency of the ROM mechanism in oblique incidence with circular polarized pulses have yielded relative efficiencies of $20 \%$ [15] or one third [16] compared to that for a linearly polarized driving pulse.

One-dimensional PIC simulations were performed using the code PICWIG [17] to investigate the suitability of transmitted CSE for polarization gating. The simulated grid contained 1000 cells per laser wavelength $(\lambda)$ and a target $\lambda / 4$ thick with a $\lambda / 10$ linear density ramp at the front

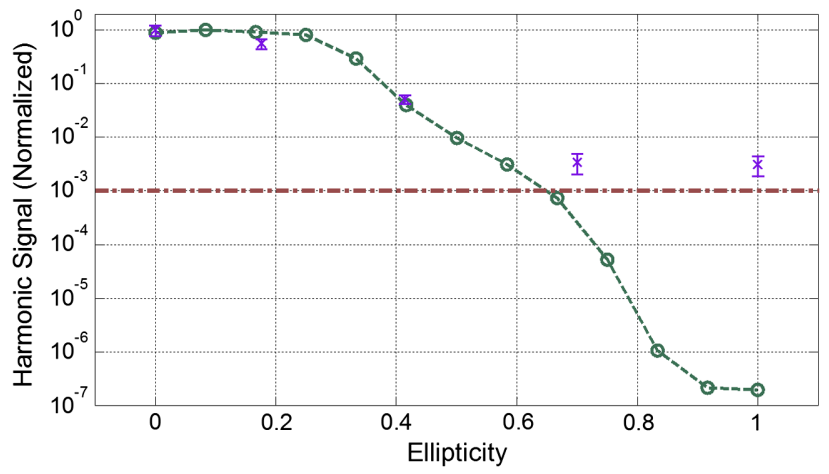

FIG. 2 (color online). Comparison of simulation and experiment. Harmonic efficiency for orders $7-15$ as obtained from PICWIG simulations (circles) is plotted alongside the integrated harmonic signal for the same orders but for the experimental data (crosses). The dash-dotted line shows the detector noise floor as obtained from a shot without a target. Data points are single shots and the errors are due to some uncertainty in the exact values used for correction of the nonharmonic background signal, while the laser energy variation was $\approx 4 \%$ for this shot set. of the target leading up to a peak density of $100 n_{c}$ where $n_{c}=\omega^{2} m_{e} \epsilon_{0} / e^{2}$ is the critical density. Ions were kept fixed and each target cell contained 1000 electron macroparticles. The interaction of an 11 cycle full width at half maximum (FWHM) pulse with peak $a_{0}=16$ was simulated for several ellipticity values to confirm that the code reproduces the experimentally observed scaling. These results are also plotted in Fig. 2. There is remarkable agreement in the observed trend for low values of ellipticity $(<0.5)$ and the simulations reproduce the same observed drop in efficiency. For higher ellipticity values the PIC code predicts several orders of magnitude more suppression than observed. This is largely due to the 1D nature of the simulations. At the intensities involved here significant denting of the target surface can be expected [1], which will create regions of oblique incidence and this effect cannot be accounted for by these simulations. Furthermore, the preplasma scale length is assumed to be the same in each case; however it is known that increasing pulse ellipticity can reduce the effect of surface heating mechanisms which may in turn affect the interaction scale length.

Polarization gating $[17,18]$ is more effective when the initial laser pulse duration is only a small number of cycles long. In this case, gating can be achieved by overlapping two colinear circularly polarized pulses with opposite handedness. In the overlap period the superposition of the two pulses results in linear polarization and the relative delay can control the duration of this linear gate. The advantage of this scheme is that it is straightforward to implement using a pair of birefringent plates [17,19]. For longer pulses the comparatively more complex IPG scheme [12] can be implemented, utilizing four pulses each with controlled timing. Two pulses are overlapped in phase so that their fields constructively add while the other two are out of phase and create a destructive minimum. One pulse pair is polarized orthogonally to the other and with a $\pi / 2$ phase shift such that their superposition is an elliptically polarized pulse where the field strengths are comparable but at the center, where only one pulse pair contributes to the total field, a linear gate is formed. The field amplitudes and variation of ellipticity are shown in Figs. 3(a) and 3(b).

Both schemes are simulated here, the former using a 2 cycle pulse with initial peak $a_{0}=12$ (corresponding to the planned capability of the LWS-20 laser [20]) while the IPG technique is simulated using an 11 cycle pulse with initial $a_{0}=16$. Simulations for both gated and ungated pulses for each method were performed, and a comparison of the plasma electron density dynamics for each scenario is shown in Figs. 3(c)-3(f).

The clearest difference in the plasma behavior is the suppression of the $2 \omega$ surface oscillations in the gated pulses for cycles outside of the linear gate (where the circular polarized laser field only drives the plasma forward). CSE is seen to be restricted to a narrow time window around the linear gate in agreement with our expectations 

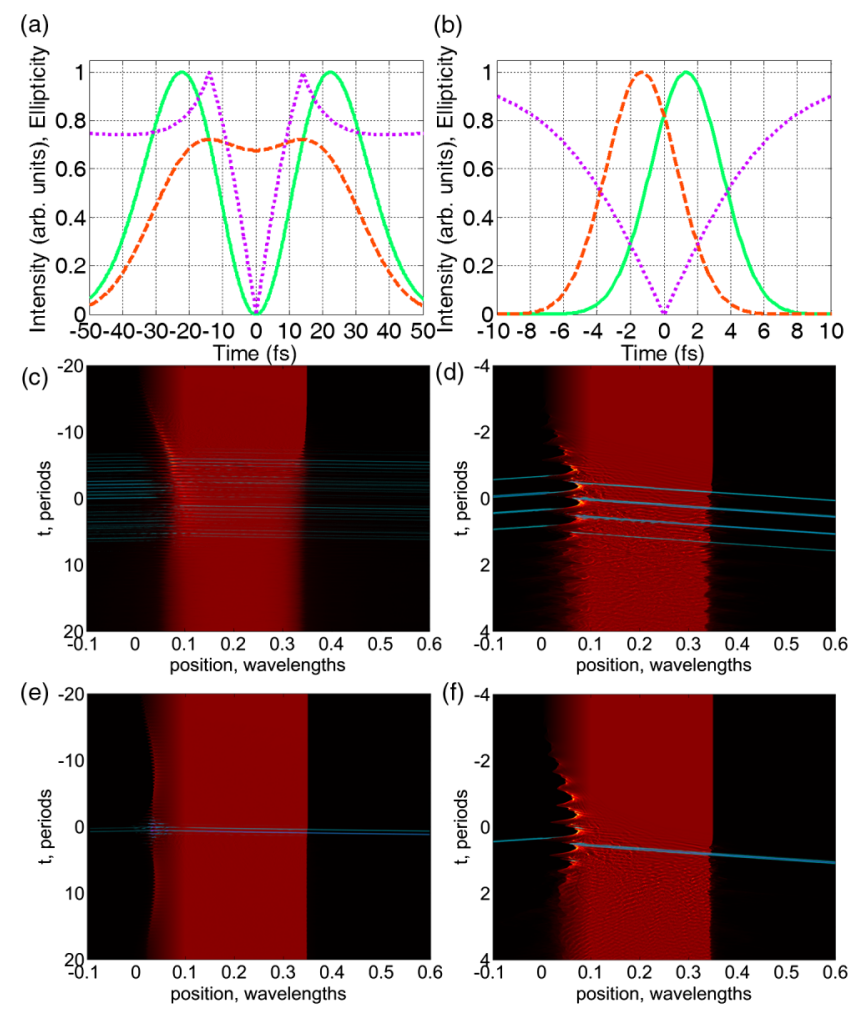

FIG. 3 (color online). Results of PICWIG simulations of polarization gating. The intensity is plotted for the constructive pulse pair (dashed) and destructive pulse pair (solid) in (a) for the IPG method. Note that these two pulse pairs are orthogonally polarized and are out of phase by $\pi / 2$. The intensities of the two opposite-handed circular pulses for the birefringent plates method are plotted in (b). In both cases the ellipticity of the composite pulse is also plotted (dotted). The electron densities from PICWIG simulations, plotted against time and position, are shown (dark areas are low density while bright regions are high) for the cases of (c) an ungated 11-cycle FWHM (full width at half maximum) Gaussian pulse (similar to the optimum capability of Astra Gemini), (d) an ungated 2-cycle pulse, (e) an 11-cycle pulse gated by IPG, and (f) a 2-cycle pulse gated by the plate method. The harmonic field intensity frequency filtered to orders above the 20th is also plotted over the density map. For the gated pulses the transmitted XUV radiation is restricted to the period of linear polarization.

based on our ellipticity dependence measurements from Fig. 2. Figure 4 plots the transmitted pulse intensity against time for each simulation. Results for the plate method, using a short 2-cycle FWHM pulse, show the clearest isolation of a single 77 as FWHM pulse with almost complete suppression of all satellite pulses. Data for the IPG method, using a longer 11-cycle FWHM pulse, show effective isolation of a single 370 as FWHM pulse with the exception of several weak satellite pulses. The large difference in the attosecond pulse duration for the two schemes is due to the different interaction conditions at the linear gate rather than as a result of the particular gating method used.
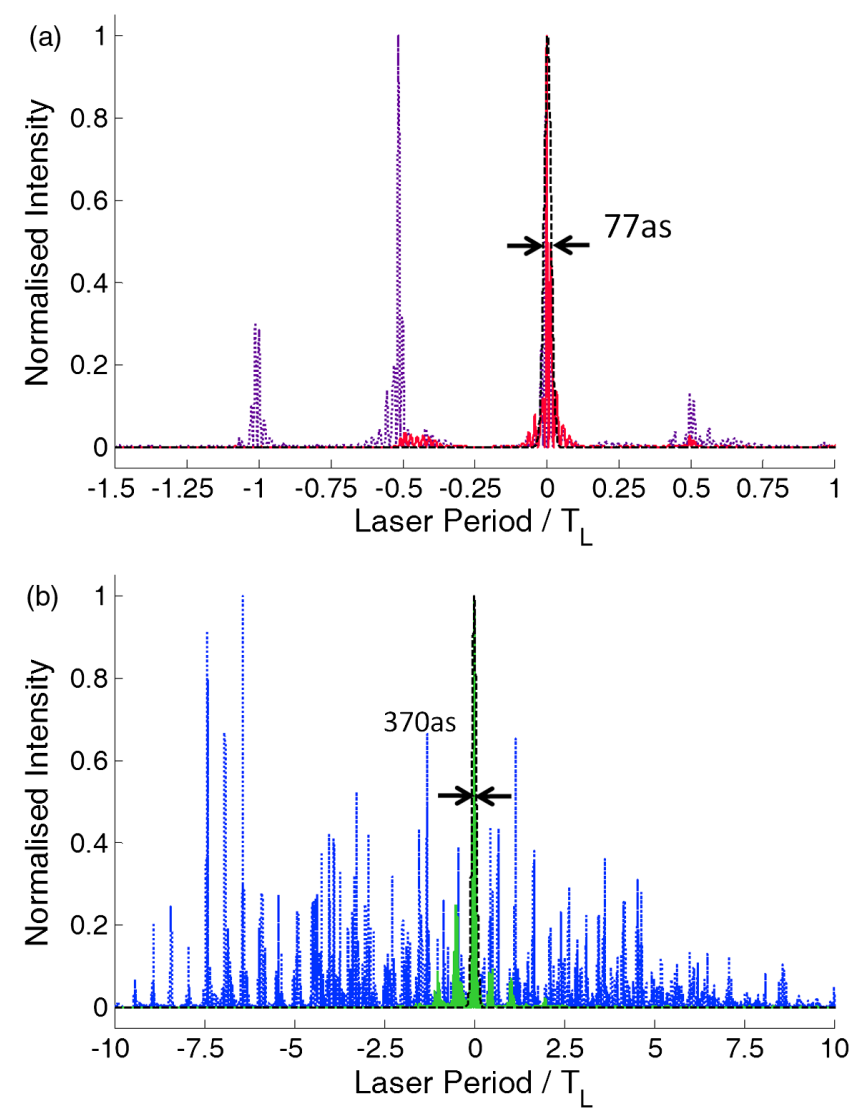

FIG. 4 (color online). Direct comparison of transmitted gated and ungated XUV pulses. (a) Plot of the normalized instantaneous intensity against time for the 2-cycle driver case for gated (solid) and ungated (dotted) pulses as obtained from PICWIG simulations. The intensity is determined by the square of the transmitted electric field after frequency filtering out harmonic orders below the 20th. Also shown is a Gaussian fit (dashed) with a FWHM of 77 as to approximate the envelope of the isolated pulse. (b) Plot for the 11-cycle pulse showing the gated (solid) and ungated (dotted) intensities. A Gaussian fit (dashed) is also shown that yields a FWHM duration of 370 as.

These results show the potential of few cycle, ultraintense pulses to produce isolated, bright attosecond XUV pulses. The high laser pulse energy of ultraintense lasers combined with considerable efficiency implies that current limits on the intensity of individual attosecond pulses in gaseous media can be overcome. Note that the efficiency and burst duration in CSE depend sensitively on laser intensity and preplasma conditions as shown by an der Brügge and Pukhov [6] for CSE in reflection. Similar behavior is observable in simulations for the transmission case and a detailed analysis will be the subject of a future publications.

In conclusion, experimental studies of the CSE process in transmission of thin foils have shown that the process can be heavily suppressed by increasing the pulse ellipticity. The possibility of exploiting this ellipticity dependence using the polarization gating technique was explored using 
1D PIC simulations and the results show that isolated, intense attosecond pulses can be generated from ultrahigh intensity interactions with thin foils.

The authors acknowledge the support of the Astra Gemini staff. B. D. acknowledges support from the EPSRC through grants and a Career Acceleration Fellowship. J. H. B. acknowledges support from the DFG Cluster of Excellence Munich Centre for Advanced Photonics (MAP) and the Transregio TR18, as well as the International Max Planck Research School of Advanced Photon Science (IMPRS-APS).

*Present address: Helmholtz-Institut Jena, Fröbelstieg 3, 07743 Jena, Germany.

†b.dromey@qub.ac.uk

*Present address: Lawrence Berkeley National Laboratory, Berkeley, California 94720, USA.

[1] B. Dromey et al., Nat. Phys. 5, 146 (2009).

[2] B. Dromey et al., Phys. Rev. Lett. 99, 085001 (2007).

[3] Y. Nomura et al., Nat. Phys. 5, 124 (2008).

[4] F. Quéré, C. Thaury, P. Monot, S. Dobosz, Ph. Martin, J.-P. Geindre, and P. Audebert, Phys. Rev. Lett. 96, 125004 (2006)
[5] R. Lichters, J. Meyer-ter-Vehn, and A. Pukhov, Phys. Plasmas 3, 3425 (1996).

[6] D. an der Brügge and A. Pukhov, Phys. Plasmas 17, 033110 (2010).

[7] B. Dromey et al., Nat. Phys. 8, 804 (2012).

[8] F. Krausz and M. Ivanov, Rev. Mod. Phys. 81, 163 (2009).

[9] P. B. Corkum, Phys. Rev. Lett. 71, 1994 (1993).

[10] P. B. Corkum, N. H. Burnett, and M. Y. Ivanov, Opt. Lett. 19, 1870 (1994).

[11] B. Dromey et al., New J. Phys. 15, 015025 (2013).

[12] P. Tzallas, E. Skantzakis, C. Kalpouzos, E. P. Benis, G. D. Tsakiris, and D. Charalambidis, Nat. Phys. 3, 846 (2007).

[13] X. Feng et al., Phys. Rev. Lett. 103, 183901 (2009).

[14] K. I. Popov, V. Yu Bychenkov, W. Rozmus, R. D. Sydora, and S. S. Bulanov, Phys. Plasmas 16, 053106 (2009).

[15] P. Heissler et al., Appl. Phys. B 101, 511 (2010).

[16] J. H. Easter, J. A. Nees, B. X. Hou, A. Mordovanakis, G. Mourou, A. G. R. Thomas, and K. Krushelnick, New J. Phys. 15, 025035 (2013)

[17] S. G. Rykovanov, M. Geissler, J. Meyer-ter-Vehn, and G. D. Tsakiris, New J. Phys. 10, 025025 (2008).

[18] T. Baeva, S. Gordienko, and A. Pukhov, Phys. Rev. E 74, 065401 (2006).

[19] I. J. Sola et al., Nat. Phys. 2, 319 (2006).

[20] P. Heissler et al., Phys. Rev. Lett. 108, 235003 (2012). 